\title{
Effects of Surface Modification, Carbon Nanofiber Concentration, and Dispersion Time on the Mechanical Properties of Carbon-Nanofiber-Polycarbonate Composites
}

\author{
Yong Gao, ${ }^{1}$ Peng He, ${ }^{1}$ Jie Lian, ${ }^{2}$ Mark J. Schulz, ${ }^{3}$ Jiang Zhao, ${ }^{1}$ Wei Wang, ${ }^{1}$ Xiaqin Wang, ${ }^{4}$ \\ Jing Zhang, ${ }^{5}$ Xingping Zhou, ${ }^{6}$ Donglu Shi ${ }^{1}$ \\ ${ }^{1}$ Department of Chemical and Materials Engineering, University of Cincinnati, Cincinnati, Ohio 45221 \\ ${ }^{2}$ Department of Nuclear Engineering and Radiological Science, University of Michigan, Ann Arbor, Michigan 48109 \\ ${ }^{3}$ Department of Mechanical Engineering, University of Cincinnati, Cincinnati, Ohio 45221 \\ ${ }^{4}$ College of Material Science and Engineering, Donghua University, Shanghai 200051, People's Republic of China \\ ${ }^{5}$ College of Science, Donghua University, Shanghai 201620, People's Republic of China \\ ${ }^{6}$ Institute of Biological Sciences and Biotechnology, Donghua University, North Renmin Road 2999, Shanghai 201620, \\ People's Republic of China
}

Received 22 November 2005; accepted 19 April 2006

DOI 10.1002/app.25112

Published online in Wiley InterScience (www.interscience.wiley.com).

\begin{abstract}
The time effect of ultrasonication was investigated for dispersing carbon nanofibers (CNFs) into a polycarbonate (PC) matrix on the mechanical properties of thusproduced composites. The effects of CNF surface modification by plasma treatment and the CNF concentration in composites on their mechanical properties were also explored. The plasma coating was characterized by HRTEM and FT-IR. Furthermore, the plasma polymerization $(10 \mathrm{w})$ treatment on the CNF enhanced the CNF dispersion in the polymer matrix. The mechanical properties of the CNF-PC
\end{abstract}

composites varied with the dispersion time, at first increasing to a maximum value and then dropping down. After a long ultrasonic treatment $(24 \mathrm{~h})$, the properties increased again. At a high concentration, the CNF-PC suspension became difficult to disperse. Additionally, the possible mechanisms for these behaviors are simply proposed. (c) 2006 Wiley Periodicals, Inc. J Appl Polym Sci 103: 3792-3797, 2007

Key words: composites; carbon nanofiber (CNF); dispersion; mechanical properties; polycarbonates

\section{INTRODUCTION}

Carbon nanotubes (CNTs) have been attracting wide interests since being discovered by S. Iijima in $1991{ }^{1}$ Due to their unique physical properties, carbon nanotubes have many potential applications. Foreseeable CNT-polymer composite materials for structural or functional applications include the field emitter, ${ }^{2}$ the tip for scanning probe microscope $(\mathrm{SPM})^{3}$, and nanotube actuators. ${ }^{4}$ However, unmodified CNTs are subject to aggregation into packed ropes or entangled networks owing to the strong intertube Van der Waals attraction, which acts as an obstacle to most applications. Especially, the aggregates of nanotube bundles effectively reduce the aspect ratio (length/diameter) of the reinforcement used in composites. Basically, two separate routes, including functionalization and ultrasonication, have been followed to disperse CNTs. Functionalization

Correspondence to: X. Zhou (xpzhou@dhu.edu.cn).

Journal of Applied Polymer Science, Vol. 103, 3792-3797 (2007) C 2006 Wiley Periodicals, Inc.

\section{(\$ILEY}

InterScience. of CNTs has shown to be effective in dispersion..$^{5-6}$ Ultrasonication is one of the main methods used to disperse the CNTs. ${ }^{7}$

Although ultrasonication of carbon nanotubes in organic solvents ${ }^{8-10}$ is widely used, the dispersion mechanism and the factors that optimize its efficiency are poorly understood. There is no detailed procedure to optimize the dispersion of surface modified nanotubes with different CNT concentrations. Nanoglues or uniform ultra-thin coatings on CNTs have been used to provide a strong link to increase the bonding strength on the CNT-matrix interface. The different coatings can be tailored to change the mechanical, electrical, and/or magnetic properties of CNTs due to a combination of changes in dimension and interface property. Recently, our group used plasma polymerization to coat carbon nanotubes and obtained a good result for the CNT dispersion in a polymer matrix. ${ }^{11}$ These surface-modified nanotubes were embedded into a polystyrene matrix and they reinforced it.

Many types of carbon nanotubes exist and the terminology is not universal. Carbon nanofiber $(\mathrm{CNF})$ usually has a larger diameter and is longer in 
(A)

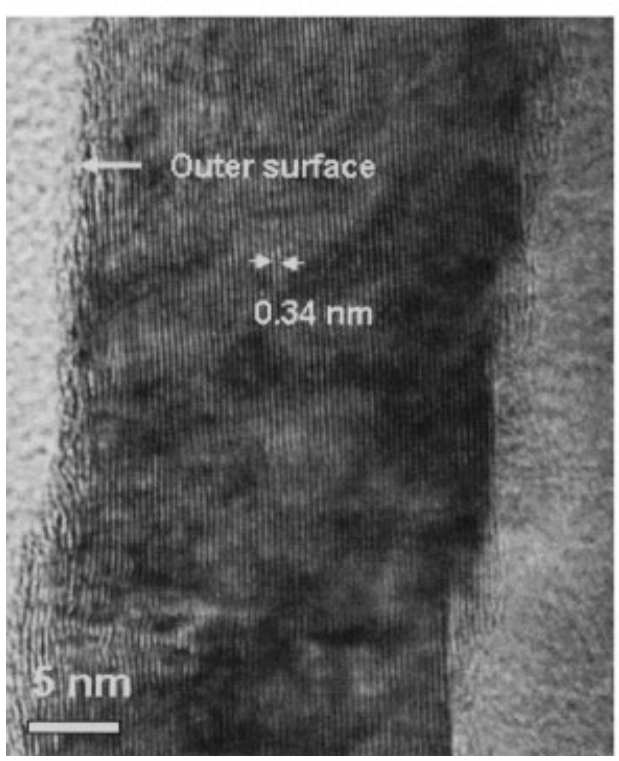

(B)

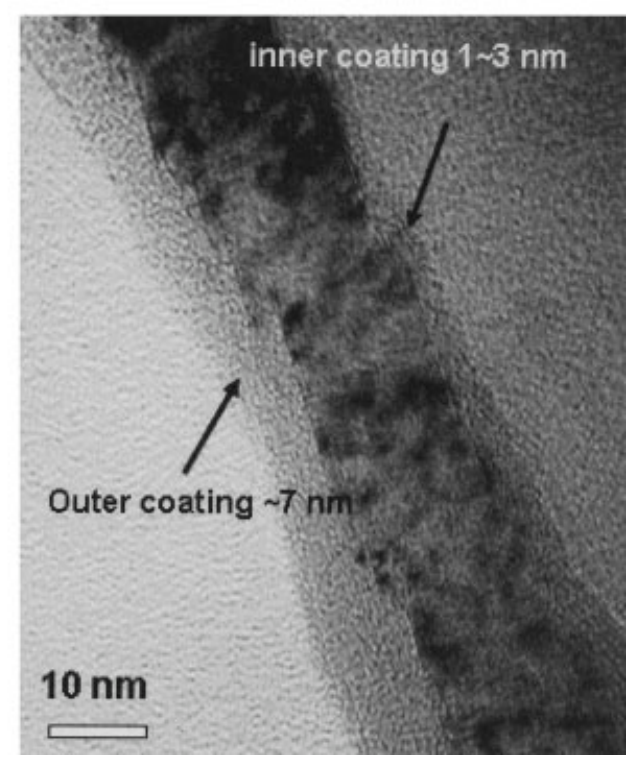

Figure 1 HRTEM images of nanofibers: (a) uncoated CNF; (b) coated CNF.

length and lower in cost than the multiwalled carbon nanotube (MWNT). In this article we address the dispersion of untreated and plasma polymerizationtreated CNFs with different concentrations into a polycarbonate (PC) matrix by ultrasonication. The focus is mainly on the dependence of the mechanical properties on the dispersion time and the CNF concentration in the CNF-PC composites. The CNF used in this research has the similar structure as MWNT so the result may give the insight of the mechanical properties of CNT-polymer composites.

\section{EXPERIMENTAL}

CNFs (Pyrograft PR 24) in size from 60 to $150 \mathrm{~nm}$ were purchased from Applied Science Inc., Cedarville, $\mathrm{OH}$. Styrene (99.5 wt \%) was obtained from Alfa Aesar, a Johnson Matthey company, Ward Hill, MA. The styrene was used to coat the CNF. Polycarbonate Resin was purchased from Fisher Scientific Inc., Chicago IL, with MW 64,000.

The CNFs were coated with a stirred plasma reactor for $1 \mathrm{~h}$ under $10 \mathrm{w}$ of plasma power. The conditions are the same as those described by Shi et al. ${ }^{11}$ The polystyrene coating was characterized using high-resolution transmission electron microscopy (HRTEM) and infrared spectroscopy (BIO-RAD FTS40 FTIR spectrometer with a BIO-RAD transmittance attachment).

A simple solution-evaporating method was employed by sonication to prepare CNF-polycarbonate composites. Two grams of polycarbonate (polymer matrix) were weighed and put into a beaker. Meanwhile, 1, 2, 3, and $5 \mathrm{wt} \%$ uncoated and coated CNFs were also put into other beakers, respectively. Chloroform was added to these beakers which separately contained the polycarbonate and CNFs. These beakers were then put into an ultrasonic tank (L\&R Solid Ultrasonic T-14B, Misawa Inc.) with $95 \mathrm{w}$ power and $23 \mathrm{KHz}$ frequency in order to disperse their contents. After the polycarbonate was dissolved completely, the solutions containing CNFs were mixed with the solutions containing polycarbonate, respectively. After ultrasonic treatment for a fixed time $(0.5,1,2,4,8,12$, and $24 \mathrm{~h})$, these mixed solutions were poured into respective aluminum molds. After the chloroform was evaporated, composite films were formed.

The films were removed from the molds and cut into several slips with dimensions of $50 \times 6 \times 0.4 \mathrm{~mm}$ for measurement of the tensile properties according to the ASTM D 822-97 (Standard Test Method for Tensile Properties of Thin Plastic Sheeting). An Instron mechanical testing machine, model 2525-818, with a speed of $1 \mathrm{~mm} / \mathrm{min}$ crosshead was used for the tensile test.

\section{RESULTS AND DISCUSSION}

The HRTEM image [Fig. 1 (a)] of the original CNF shows the graphite structure with interlayer spacing $d_{002}=0.34 \mathrm{~nm}$. Parallel graphite layers are clearly seen. The shell thickness of the CNF can be estimated to be about 20-30 nm. Figure 1(b) shows the HRTEM image of the plasma surface-modified CNF. 


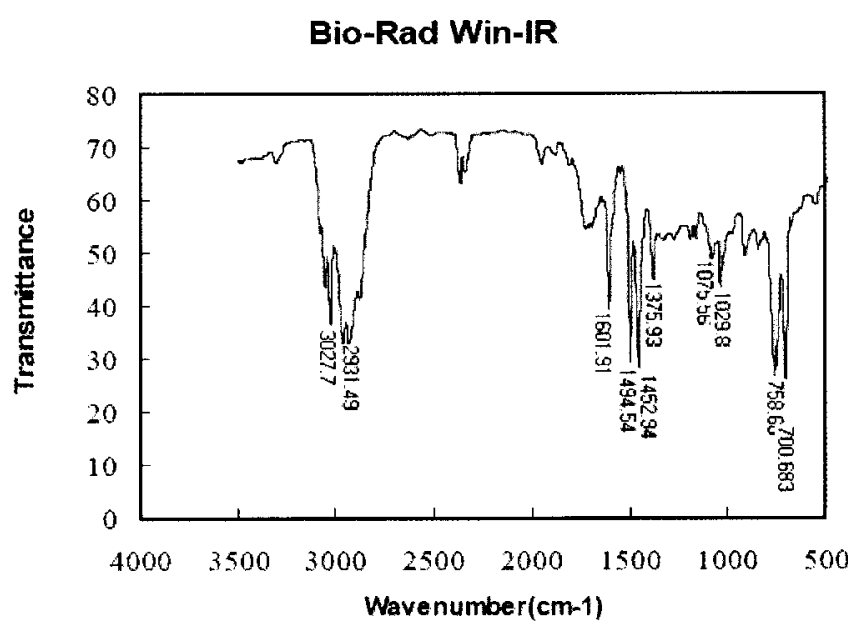

Figure 2 FTIR spectrum of the polystyrene coated CNF.

Ultrathin thin films of amorphous layers can be clearly seen covering the both side of CNF shell. The thin films are uniform with thickness of approximately $7 \mathrm{~nm}$ for the outing coating and 1-3 $\mathrm{nm}$ for the inner coating.

In order to further investigate the surface structure of the films on CNFs, FT-IR was conducted; the spectrum is shown in Figure 2. The characterization peaks of FI-IR can be found in the study by Kuptsov and Zhizhin. ${ }^{12}$ The spectrum exhibits several very strong peaks between 1400 and $1600 \mathrm{~cm}^{-1}$, which result from the $\mathrm{C}-\mathrm{C}$ vibrating absorption of the benzene ring. The several peaks are due to the special absorption of the benzene ring. These peaks confirmed that the plasma polymer structure contained some benzene rings. In addition, the peaks in the wave number ranging from 690 to $900 \mathrm{~cm}^{-1}$ belong to the benzene $\mathrm{C}-\mathrm{H}$ out-of-plane bending. Actually in Figure 2, at 758 and $700 \mathrm{~cm}^{-1}$, these two peaks are due to the special absorption for one hydrogen atom of a benzene ring substituted with other functional groups. Although the standard FT-IR spectrum of polystyrene is relatively simple in comparison to that shown in Figure 2, the special absorption peaks for styrene are almost the same as those for common polystyrene. This reveals that in plasma polymerization the molecular structure is destroyed by ion or electron bombardment. However, the deposited film still maintains some polystyrene molecular structure, which is desirable for compatibility with the polycarbonate matrix.

For dispersion time from 0.5 to $12 \mathrm{~h}$, the tensile strength of the CNF-PC composites of different CNF concentrations had a similar trend (see Fig. 3). First, the tensile strength increases with the dispersion time and then reaches a maximum value and decreases continuously. The Young's modulus had a similar trend as the tensile strength (Fig. 4). Ultraso- nication imparted a very high energy density into the dispersion. The mechanism of deagglomeration lies in cavitation effects arising adjacent to particles or within loosely associated particle aggregates and the inability to follow the induced wave front projected by the transducer. When two particles are separated by a distance of only a few particle diameters, hydrodynamic forces of repulsion and attraction are involved in the ultrasound field. ${ }^{13}$ The maximum tensile strength is the balance status of the contracting and repelling forces created in the ultrasound field. The results also showed that the separation of the nanotube from the bundle was reversible, leading to a dynamic equilibrium for the system of the surfactant adsorption during ultrasonication. ${ }^{14}$ The decrease in the tensile strength after maximum value may come from the decrease in the ultrasound velocity. The study by Geza and Harald ${ }^{15}$ showed that the sound velocity decreased with the dispersion time and then jumped again for a long dispersion time.

Generally, the composites with coated CNFs have higher tensile strength than that of uncoated CNFs. This indicates, in turn, that the coated CNFs are better dispersed than the uncoated CNFs. The tensile strength of composite with uncoated CNF can not exceed the blank PC. Figure 5 shows the SEM image of the uncoated CNF dispersed by ultrasonication for $2 \mathrm{~h}$. Figure 6 shows the SEM images of the composites with different concentrations of the coated CNF at their optimum dispersion status: 1 wt \% coated CNF $(10 \mathrm{w})$ dispersed for $2 \mathrm{~h}$ (above) and $5 \mathrm{wt} \%$ coated CNF (10 w) dispersed for $8 \mathrm{~h}$ (below). Figure 5 (compared to Fig. 6) displayed that the uncoated CNF had carbon fiber clusters, and large pores were found in the polymer even under the optimum ultrasonication condition. That means CNF cannot give good mechanical properties, even under the optimum dispersion condition. Lau and Hui ${ }^{16}$ reported that the use of multiwalled nanotubes for advanced composite structures did not improve the mechanical strength of the structure. The weak bond between the outer shell of the CNF and polymer matrix may contribute to this; it only affects the outer shells of the nanotubes. The inner shells can rotate and slide freely due to the weak Van der Waals force among the individual shells. This results in the nonuniform axial deformation inside the multiwalled nanotubes.

At the maximum tensile strength, the $1 \%$ and $2 \%$ composites have higher tensile strength than that of the pure PC polymer. The tensile strength of 3 and $5 \mathrm{wt} \%$ composites did not exceed the value of the pure PC polymer. This means that the composites with higher concentration of CNF are difficult to be dispersed well using sonication. The greatest increase in tensile strength for $1 \mathrm{wt} \% \mathrm{CNF}$ coated at 

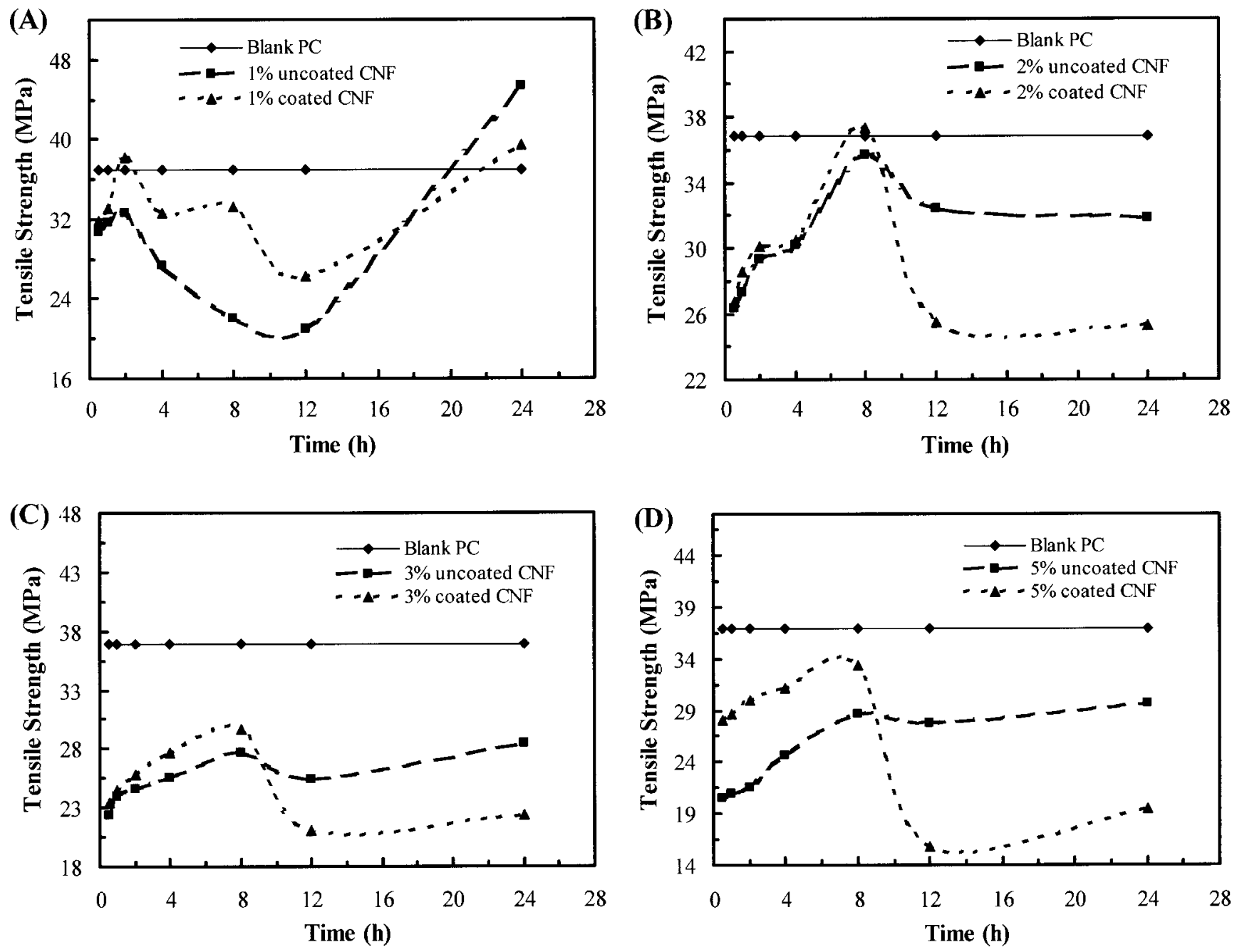

Figure 3 Tensile strength of composites with different CNF concentrations changes with ultrasonication time.

$10 \mathrm{w}$ plasma power is only $3.5 \%$ in comparison to the pure PC polymer.

For composites with different concentrations of $\mathrm{CNF}$, the time to reach the maximum strength is different: it is $2 \mathrm{~h}$ for the $1 \mathrm{wt} \%$ composites and $8 \mathrm{~h}$ for the other composites. This means that longer time is required to disperse composites with a high concentration of CNF than with a low concentration. This is because of the limited space for the movement of the CNF in polymer matrix. Figure 6 showed that $5 \mathrm{wt} \%$ coated CNF at its optimum dispersion distributed more randomly than the $1 \mathrm{wt} \%$ coated CNF.

For the overnight dispersion time ( $24 \mathrm{~h}$ ), the tensile strength showed a different trend. Basically, the tensile strength of the uncoated and coated CNF-PC composites increases to some extent. For the composite with $1 \mathrm{wt} \%$ uncoated CNF dispersed after $24 \mathrm{~h}$, the maximum tensile strength increased $23 \%$ over the pure PC film. But that of the composite with $1 \%$ coated CNF only increased $6.7 \%$. This change may result from the change in the velocity of the ultrasound. As described above ${ }^{15}$ in the suspension system, the velocity of ultrasound decreased with the dispersion time and jumped to a high velocity again for a long dispersion time. The uncoated CNF-PC composites usually show a better tensile strength after a longer dispersion time. The mechanism is still not clear. According to Lu et al., ${ }^{17}$ long time ultrasonication damages the CNFs. The destruction of CNFs seems to initiate on the external layers and travel towards the center. The study also showed that the nanotube layers seemed quite independent, so the CNFs may not get shorter, but actually became thinner with time. With the decrease in the layer number of the $\mathrm{CNF}$, the size of nanofibers becomes smaller. Another possible reason for the coated CNF-PC composites with lower mechanical properties than the uncoated counterpart after longtime treatment may arise from the follow process. The coating dissociates into the radicals after a long time of ultrasonication. The resident radicals may 

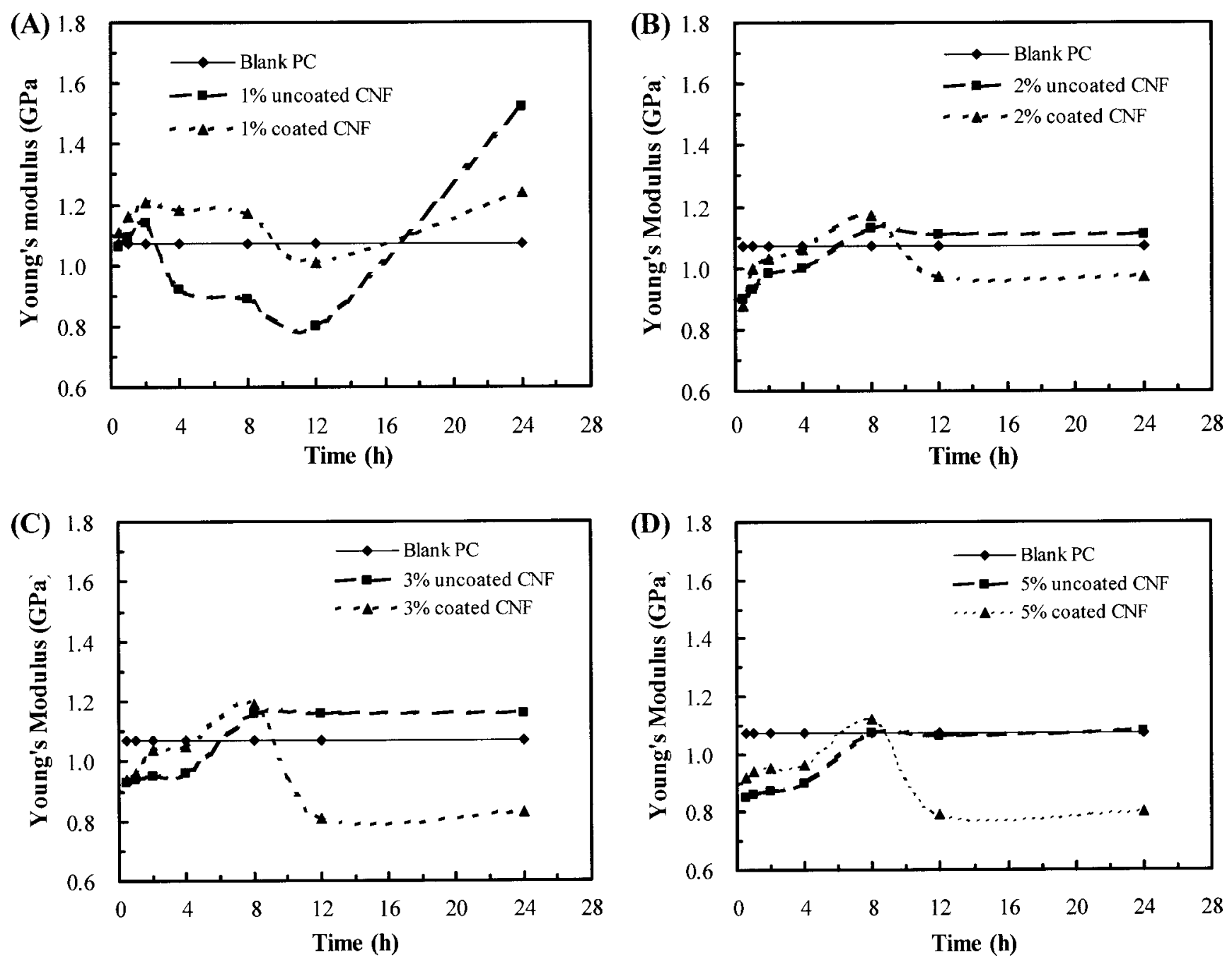

Figure 4 Young's modulus of composites with different CNF concentrations changes with the ultrasonication time.

link again to form the agglomeration locally which contributes to the lower tensile strength than that of composite with uncoated CNF after a long dispersion time. This mechanism has not been verified and more experiments need to be done in a future work to confirm this. For the low CNF concentration ( $1 \mathrm{wt} \%$ $\mathrm{CNF}$ ), the increase is relatively large. The increasing amplitude of high-concentration CNF-PC composites $(2,3$, and 5 wt \%) showed a lower increase or was nearly unchanged. This trend also reflected the fact that high-concentration CNF is difficult to disperse.

The Young's modulus has the similar trend as the tensile strength (Fig. 4). Before $12 \mathrm{~h}$ dispersion, the highest Young's modulus increased $13 \%$ above the modulus of the blank PC for the composite with $1 \mathrm{wt} \%$ coated CNF. After $24 \mathrm{~h}$ dispersion, the maximum Young's modulus increases $42 \%$ above the modulus of the blank PC for the composite with 1 wt \% uncoated CNF. These results can be explained according to the same mechanisms.

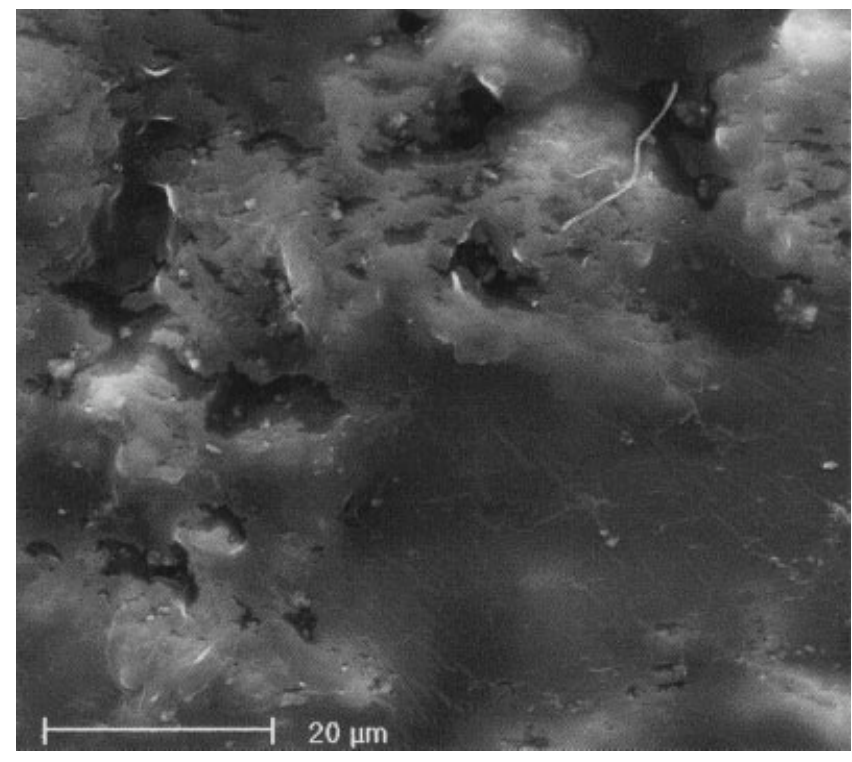

Figure 5 SEM image of the composite embedded with the 1 wt \% uncoated CNF dispersed for $2 \mathrm{~h}$. 

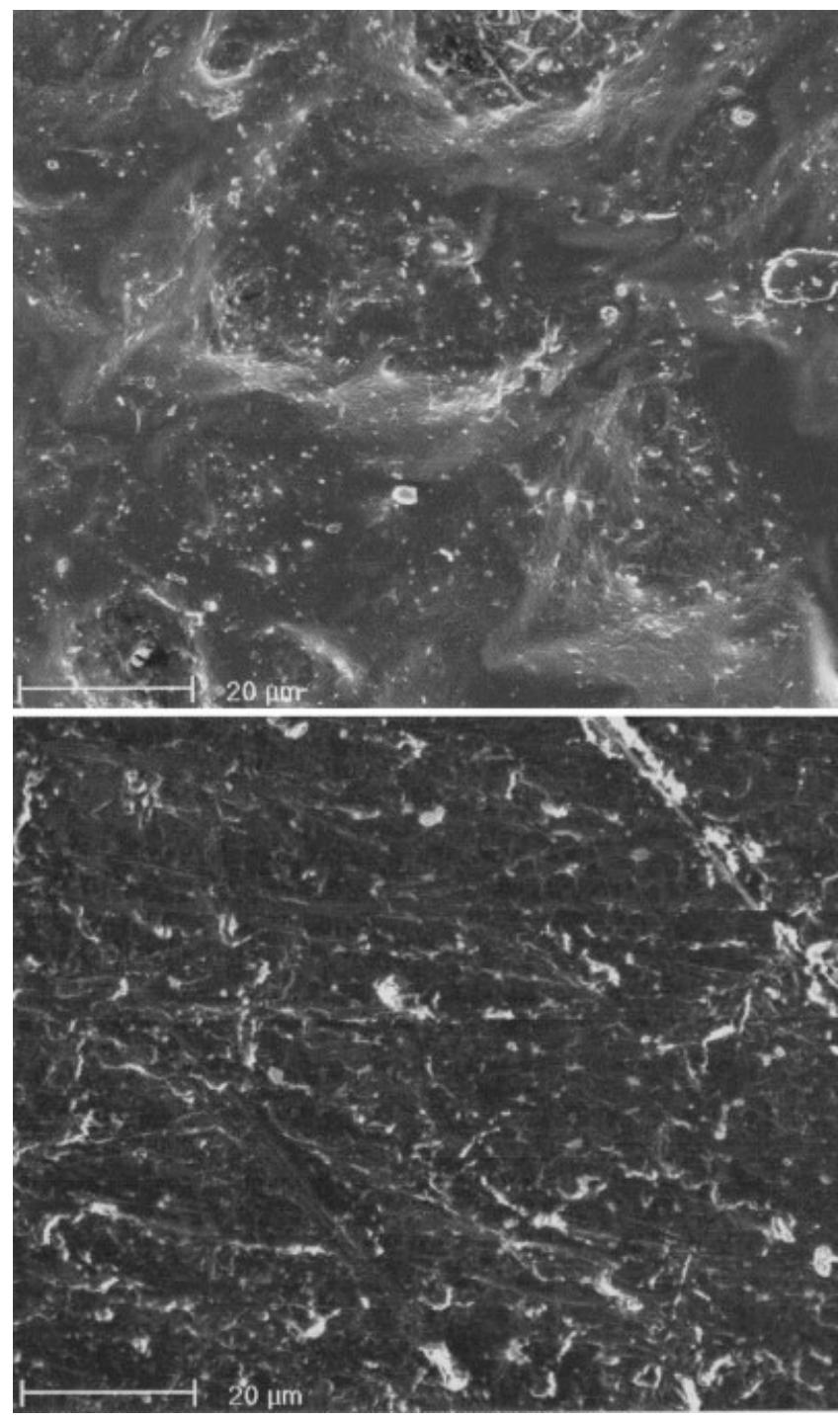

Figure 6 SEM images of the composites embedded with different concentrations of the coated CNF at their optimum dispersion status: $1 \mathrm{wt} \%$ coated CNF dispersed for $2 \mathrm{~h}$ (above); $5 \mathrm{wt} \%$ coated CNF dispersed for $8 \mathrm{~h}$ (below).

\section{CONCLUSIONS}

Based on above the data and discussion, the following conclusions can be drawn:

1. The plasma polymerization-treated CNFs showed a better dispersion than that for pure CNFs. This showed, in turn, that treated CNFs had a greater tensile strength and Young's modulus than the pure CNFs dispersed in the PC matrix, except for overnight dispersion.

2. The tensile strength and Young's modulus of CNF-PC composites depended on the CNF dis- persion in the PC matrix. The mechanical properties increased with the dispersion time and reached a maximum as the balance of contracting and repelling forces was reached, and then the properties decreased with the dispersion time. For overnight dispersion, the mechanical properties again increased somewhat.

3. The ultrasonication time to reach the best dispersion increased with the CNF concentration. It is more difficult to disperse the CNFs with increasing $\mathrm{CNF}$ concentration in the polymer matrix.

4. The possible reason for the increase of the tensile strength and the Young's modulus may be the increase in ultrasound velocity, the peeling of the outer layers of the CNFs, and the change in the surface status of the CNFs.

\section{References}

1. Iijima, S. Helical microtubules of graphitic carbon. Nature 354, 1991, 56

2. Saito, Y.; Uemura S. Carbon 38, 2000, 169.

3. Dai, H. J.; Jason, H. H.; Andrew, G. R.; Daniel, T. C.; Richard, E. S. Nature 384, 1996, 147.

4. Ray, H. B.; Cui, C. X.; Anvar, A. Z.; Zafar, I.; Joseph, N. B.; Geoff, M. S.; Gordon, G. W.; Alberto, M.; Danilo, D. R.; Andrew, G. R.; Oliver, J.; Siegmar, R.; Miklos, K. Sci 284, 1999, 1340.

5. Sun, Y. P.; Fu, K. F.; Lin, Y.; Huang, W. J. Acc Chem Res 35, 2002, 1096.

6. Chen, J.; Rao, A. M.; Lyuksyutov, S.; Itkis, M. E.; Hamon, M. A.; Hu, H.; Cohn, R. W.; Eklund, P. W. J Phys Chem B 200, 105, 2525.

7. Liu, J.; Casavant, M. J.; Cox, M.; Walters, D. A.; Boul, P.; Lu, W.; Rimberg, A. J.; Smith, K. A.; Colbert, D. T.; Smalley, R. E. Chem Phys Lett 303, 1999, 125.

8. Koshio, M.; Yudasaka, T.; Zhang, M.; Iijima, S. Nano Lett 2001, $1,361$.

9. Hou, P. X.; Liu, C.; Tong, Y.; Xu, S. T.; Liu, M.; Cheng, H. M. J Mater Res 16, 2001, 2526

10. Hou, P. X.; Bai, S.; Yang, Q. H.; Liu, C.; Cheng, H. M. Carbon 2002, 40, 81.

11. Shi, D. L.; Lian, J.; He, P.; Wang, L. M.; Xiao, F.; Yang, L.; Schulz, M. J.; David, B. M. Appl Phys Lett 83, 2003, 5301.

12. Kuptsov, A. H.; Zhizhin, G. N. Handbook of Fourier Transform Raman and Infrared Spectra of Polymers; Elsevier Science: The Nertherlands, 1998, ch. 11.

13. Saad, M. A. Compressible fluid flow; Prentice Hall: Englewood Cliffs, N.J. 1993, 60

14. Michael, S. S.; Valerie, C. M.; Michael, K. M.; Mathew, J. A.; Erik, H. H.; Carter, K.; Robert, H. H.; Smalley, R. E. J Nanosci \& Nanotech 3, 2003, 81.

15. Geza, H. S.; Harald, H. J Colloid \& Interface Sci 189, 1997, 123.

16. Lau, K.T.; David, H. Carbon 40, 2002, 1605.

17. Lu, K. L.; Lago, R. M.; Chen, Y. K.; Green, M. L. H.; Harries, P. J. F.; Tsang, S. C. Carbon 34, 1996, 814. 